\title{
A Novel Multilayer Perceptron Artificial Neural Network based Recognition for Kurdish Manuscript
}

\author{
Behnam Zebardast ${ }^{*}$, Isa Maleki ${ }^{2}$ and Awat Maroufi ${ }^{3}$ \\ 1,3Department of Computer, Boukan Branch, Islamic Azad University, Boukan, Iran; \\ behnamzebardast@gmail.com, awat.maroofi@gmail.com \\ 2Department of Computer Engineering, Dehdasht Branch, Islamic Azad University, \\ Dehdasht, Iran; maleki@iaudehdasht.ac.ir
}

\begin{abstract}
Alphabetic recognition is one of the most interesting and successful research fields in artificial intelligence and pattern recognition. The different writing structures of the languages and the presence of different approaches in diagnosis of letters of the different languages has been a challenge in alphabetic recognition. All these challenges have made many researches switch this research area. Of the different approaches in alphabetic recognition, the neural artificial networks could have been successful according to the capability of parallel process and learning capability for a special application like recognition pattern. So, it is a suitable approach in alphabet recognition. The Kurdish language holds two manuscripts according to Latin and Arabic alphabets. In this paper the Multilayer Perceptron (MLP) artificial neural networks are studied by the back-propagation algorithm to recognize the Kurdish-Latin manuscript. The proposed method is also applicative for diagnosis of all letters of the other Latin languages like English, Italian and etc. In this paper the MLP artificial neural networks are implemented in MATLAB environment. The efficiency factor for recognition of the Kurdish letters is to maximize the recognition accuracy of the Kurdish letters in training and testing the MLP artificial neural networks. This accuracy is $85.1535 \%$ in training stage and $81.2677 \%$ in testing stage.
\end{abstract}

Keywords: Accuracy of Testing, Accuracy of Training, Kurdish Letters Recognition, Multilayer Perceptron Artificial Neural Networks

\section{Introduction}

Many important steps have been taken for identifying the pattern in late decades. A pattern can be a letter or a sample manuscript, a goal for radar, speaking signal, cardiograph signal, signature, fingerprint or even a part of the parts of production line in a factory ${ }^{1,2}$. It could be seen that this field is very vast. So the letters diagnosis could be a subject in identifying the patterns as interesting research fields for the researchers. Although there are diverse kinds of languages which leads to the challenges in letter diagnosis, the automation process of the pattern recognition machines has made this easier ${ }^{2,3}$. Reading the alphabetic symbols and the numbers is a sample of automation process of the machines in pattern recognition ${ }^{3}$. It must be said that now automation of the machines for pattern recognition leads to the serious advances in science and technology from the increasing the speed and preciseness, reducing the role of human in repetitive and tiring works, frugality in time and reducing the error rate in letters diagnosis points of view ${ }^{4}$. One of the machines which has been located in all technologies in the recent half a century, is the artificial neural networks ${ }^{4,5}$. Pattern recognition, artificial intelligence and neural modeling are common in beginning point in history as they all have been started from $1950 \mathrm{~s}^{5}$. Generally the pattern recognition is studied in many fields like artificial neural networks. So this is why artificial neural networks application is a method for strengthening the pattern recognition in comparison to the other methods and on this way, In this regard the application of the artificial neural networks for letters recognition and other kinds of patterns have

${ }^{*}$ Author for correspondence 
been proposed because the artificial neural networks can fulfill some shortages of the common pattern recognition methods and represent interesting results ${ }^{6}$.

One of the applications of the artificial neural networks in letters or numbers recognition which is seen today is reading the bank cheques in less time than human being or reading the plate number of the cars ${ }^{4}$. The letter recognition using the artificial neural networks could be very hard as it is hard to write the letters in different sizes and the writing style and transformation of the letters to matrix in different sizes are very hard. So it must be first tried to identify a specific size in transforming any letter to a matrix to make letters has a same size when transformed to the matrix one by one, and this avoids any noise and additional information, and the artificial neural networks is a good solution for it ${ }^{7}$. Artificial neural networks are a method which tries to mimic the behavior of the minds of the people which are called brain neurons and simulate them. Artificial neural networks have been used in many applications like recognition and prediction which would need the simulation tools in problems analysis ${ }^{8}$. Using the artificial neural networks models it is possible to implement and design the letters recognition complex processes in form of software. One of the applications of the artificial neural networks is that it is used for the problems and methods in which there is no algorithmic solution for or don't follow any relationship ${ }^{9,10}$. The letter recognition via artificial neural networks take place by the data training which must be repeated many times to lead good results about letter recognition ${ }^{10,11}$. Studying the literature, it could be said that the researchers have tried to recognize the letters in Persian ${ }^{7,12}$, Arabic ${ }^{7,13,14}$, English ${ }^{15,16}$, Indian languages ${ }^{17-19}$ and Chinese ${ }^{20}$ using the different models of artificial neural networks and the reason the researchers have not selected the Kurdish language is that they are no way familiar with this language. The artificial neural networks are classified in two groups feed-forward and recurrent ${ }^{21}$. Feed-Forward networks include many kinds of artificial neural networks the most important of which is the MLP artificial neural network and in this paper we have used MLP artificial neural network for Kurdish letter recognition. The efficiency factor in this method is to reach the maximum accuracy in Kurdish letters recognition in training and testing stages. The proposed method in this paper is used for the letter recognition of all other Latin languages like English, Italian and etc. In this paper we have implemented the MLP artificial neural networks in MATLAB environment.
In Section 2, we will talk about the related works done for letter recognition in different languages; In Section 3, of the paper the Latin-Kurdish letters manuscript and the recognition method of them is studied; In Section 4, of the paper of MLP artificial neural networks and the proposed method for this network is introduced. And, finally the Section 5, will be conclusion and future works discussed.

\section{Related Works}

Kurdish language holds two common manuscripts based on Latin and Arabic alphabet and no model of artificial neural networks has been used for none of the two Kurdish manuscripts in letter diagnosis. But all of the researches are based on letters recognition of English, Arabic, Persian and Indian languages like Tamil, Telugu and etc. In this paper we have introduced the related works in the field of recognition of the English, Arabic, Persian and Indian languages letters with different models of artificial neural networks and have tried to make the researchers and the readers of this paper clearly understand the letters diagnosis of the different languages using artificial neural networks models so it is not possible to compare the works about the none Latin- Kurdish letters recognition to the results of this paper which is about the recognition of the Kurdish letters via MLP artificial neural networks. From this point of view in this section (related works), in the first part we will talk about the previous researches in recognition of the Persian and Arabic and Hindi letters by MLP artificial neural networks and the previous researches in recognition of the letters of the different languages by the different artificial neural networks in the second part.

\subsection{Recognition of the Different Languages Letters by the MLP Artificial Neural Networks}

Researchers ${ }^{18}$ have used MLP artificial neural networks using the back-propagation algorithm and the Radial Basis Function (RBF) artificial neural networks for recognition of the Hindi letters. In this method a dataset which includes 245 repetitive characters of Hindi letters is used in which 125 characters are used as training data (training set) and 120 characters are used as testing data (testing set). In this method the results showed that the MLP artificial neural networks using learning back-propagation algorithm was better in recognition accuracy and memory usage. 
In another research ${ }^{7}$ the MLP artificial neural networks are used for recognition of the Arabic and Persian manuscript in which the back-propagation algorithm was used for MLP learning. The MLP artificial neural network holds three layers. In this method there are 30 neurons in the input layer and 11 neurons in hidden layer and 4 neurons in output layer. In this method 10 characters are selected from Persian and Arabic manuscript and 50 handwriting sample of any letter is present all of which are like the images in definite sizes. The training rate for 40 sample of any letter is $80 \%$ and the testing rate for 10 samples of each letter is $20 \%$. And using these rates, the Mean Square Error in any repetitions, it is tried have the least rate of error until it gets to zero.

\subsection{Recognition of the Letters of Different Languages by Different Models of Artificial Neural Networks}

In a research ${ }^{22}$, the RBF artificial neural networks in which the combination of K-Means clustering and genetic algorithms are used, are utilized for recognize the first name of the people in Persian and Arabic languages. 110 samples are used for 30 names, 100 samples of which are used for training and 10 samples are used for testing. At the utmost accuracy the recognition of these names in RBF artificial neural networks in combination to a genetic algorithm has led to the improvement of recognition of these names.

In reference ${ }^{23}$ the RBF artificial neural networks are used for recognition of handwriting of 20 writers using 500 handwritings which were written by these writers. The recognition of these manuscripts takes place using the scanned images of the texts, and the accuracy manuscript recognition of the 20 writers was 90 to 100 percent.

Researchers ${ }^{17}$ have used the Probabilistic Neural Networks (PNN) and the RBF artificial neural networks to recognize the Telugu letters which is the language of more than 100 million people in south India. In this method, 600 taken images of Telugu letters are used and the efficiency factors for these networks are accuracy, sensitivity, specificity, Positive Predictive Value (PPV) and Negative Predictive Value (NPV). Then these factors are compared to each other for the two artificial neural networks. Finally the Telugu letters recognition for RBF artificial neural networks was better than PNN artificial neural networks from efficiency factor point of view.
In another research ${ }^{24}$, the researchers did the recognition of the typed letters using Fuzzy method which is very accurate in recognition of the typed letters but is not good enough in recognition of the handwriting.

In reference ${ }^{25}$ a hybrid neural network is used for recognition of the English handwriting letters. The neural network inputs are in the form of the images of classified letters. The proposed method for this hybrid neural network is using the Self-Organizing Feature Map (SOFM) algorithm, gradient descent learning algorithms and the Multilayer Feed-forward Networks (MLFN) and they have led to the improvement of the recognition of the English letters.

Also reference ${ }^{26}$ has implemented that the recognition of the handwriting letters using the pen tip movements in his thesis which is another way of recognizing the letters. And if there is no enough accuracy in writing, the system will not be able to deal with.

It is clear from the researches in letter recognition of different languages that no study is done on recognition of the Kurdish letters with different models in any artificial neural networks. It is clear from the researches that there is lack of information in Kurdish letters recognition and this has been the inspiring factor for choosing Kurdish letters recognition as the subject of this paper.

\section{Recognition of the Kurdish Language Letters}

As it is said before the Kurdish language holds two common manuscripts according to the Latin and Arabic alphabets. In this paper the Latin-Kurdish letters are selected for recognition according to the MLP artificial neural networks. Latin-Kurdish includes 31 letters. Table 1 shows these letters.

According to the Table 1, we have created each letter of Kurdish language in the form of a matrix $7^{*} 11$ of one and zero values. Then each $7^{*} 11$ matrix was changed to $77^{*} 1$ matrix in which the numbers zero and one rows of matrix $7^{*} 11$ are tandem placed in one column. Then, each $77^{*} 1$ matrix which shows one letter of Kurdish language is placed in the

Table 1. Latin-Kurdish letters

\begin{tabular}{|c|c|c|c|c|c|c|c|}
\hline A & B & C & D & C & E & $\hat{\mathbf{E}}$ & F \\
\hline G & H & I & J & $\hat{\mathbf{I}}$ & K & L & M \\
\hline $\mathbf{N}$ & $\mathbf{O}$ & $\mathbf{P}$ & $\mathbf{R}$ & $\mathbf{Q}$ & $\mathbf{S}$ & Ş & T \\
\hline $\mathbf{U}$ & $\hat{\mathbf{U}}$ & $\mathbf{V}$ & $\mathbf{X}$ & $\mathbf{W}$ & $\mathbf{Y}$ & $\mathbf{Z}$ & \\
\hline
\end{tabular}


general matrix which is named alphabet matrix which is in $77^{*} 31$ dimensions. The number 77 shows that each letter is in form of $77^{*} 1$ matrix and number 31 shows that there are 31 letters in Kurdish Language. The goal of this is to collect all Kurdish letters in a general matrix named Alphabet. For example the Kurdish letter Î which is in form of a $7^{*} 11$ matrix, is showed in Figure 1 and 2.

Figure 1 which is the letter $\hat{\mathrm{I}}$ is cited in this paper to make the Figure 2 which is the $7^{*} 11$ matrix of letter $\hat{I}$ be understood and shown clearly and we have transformed all 31 letters of Kurdish language to the $7^{*} 11$ matrixes one by one and of course the $7^{*} 11$ dimension is completely optional for each letter and we can select the other dimension. Of course according to the experiments on MLP, the more the matrix dimension, the longer will be the MLP learning.

According to the Figure 2, we save the $7^{*} 11$ matrix of letter $\hat{I}$ in a $77^{*} 1$ matrix named Letter $\hat{I}$ matrix in which we put tandem zero and one numbers of the rows of the matrix $7^{*} 11$ in one column and the Table 2 shows the matrix $77^{*} 1$ of the Î letter.

The transformation method of each letter to $77^{*} 1$ matrixes is as the process of Figure 2 and Table 2. We have 31 matrixes of $77^{*} 1$ because there are 31 Kurdish letters. Which finally 31 matrixes of $77^{*} 1$ are put in a general matrix named Alphabet and then the Alphabet matrix is

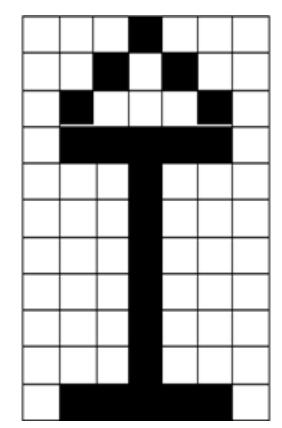

Figure 1. The image of the letter $\hat{I}$.

$$
\left[\begin{array}{lllllll}
0 & 0 & 0 & 1 & 0 & 0 & 0 \\
0 & 0 & 1 & 0 & 1 & 0 & 0 \\
0 & 1 & 0 & 0 & 0 & 1 & 0 \\
0 & 1 & 1 & 1 & 1 & 1 & 0 \\
0 & 0 & 0 & 1 & 0 & 0 & 0 \\
0 & 0 & 0 & 1 & 0 & 0 & 0 \\
0 & 0 & 0 & 1 & 0 & 0 & 0 \\
0 & 0 & 0 & 1 & 0 & 0 & 0 \\
0 & 0 & 0 & 1 & 0 & 0 & 0 \\
0 & 0 & 0 & 1 & 0 & 0 & 0 \\
0 & 1 & 1 & 1 & 1 & 1 & 0
\end{array}\right]
$$

Figure 2. Matrix $7^{*} 11$ of letter $\hat{I}$.
Table 2. Matrix Letter Î in $77^{*} 1$ dimensions

\begin{tabular}{|l|}
\hline 0 \\
\hline 0 \\
\hline 0 \\
\hline 1 \\
\hline 0 \\
\hline 0 \\
\hline 0 \\
\hline 0 \\
\hline 0 \\
\hline 1 \\
\hline 0 \\
\hline 1 \\
\hline 0 \\
\hline 0 \\
\hline 0 \\
\hline 1 \\
\hline 0 \\
\hline 0 \\
\hline 0 \\
\hline 1 \\
\hline 0 \\
\hline 0 \\
\hline 1 \\
\hline 1 \\
\hline 1 \\
\hline 1 \\
\hline 1 \\
\hline 0 \\
\hline 0 \\
\hline 0 \\
\hline 0 \\
\hline 1 \\
\hline 0 \\
\hline 0 \\
\hline 0 \\
\hline 0 \\
\hline 0 \\
\hline 0 \\
\hline 1 \\
\hline 0 \\
\hline 0 \\
\hline 0 \\
\hline 0 \\
\hline 0 \\
\hline 0 \\
\hline 1 \\
\hline 0 \\
\hline 0 \\
\hline 0 \\
\hline 0 \\
\hline 0 \\
\hline 0 \\
\hline 1 \\
\hline 0 \\
\hline 0 \\
\hline 0 \\
\hline 0 \\
\hline 0 \\
\hline 1 \\
\hline 1 \\
\hline 1 \\
\hline 1 \\
\hline 0 \\
\hline
\end{tabular}


created in the form of a $77^{*} 31$ matrix. The alphabet matrix creation method is as shown in Table 3.

According to the Table 3, we save all letters from LetterA to LetterZ any of which is in the form of a $77^{*} 1$ matrix in Alphabet matrix. At last the alphabet matrix will be created in $77^{*} 31$ dimensions. The Alphabet Matrix is the input matrix. Now to create the Target matrix and according to the 31 Kurdish letters, we create a $31 * 31$ matrix. This matrix has a 1 value in each column that placed in the row related to each number of the each letters. Table 4 shows the Target matrix. For example, A letter holds the number 1 in the first row and the other numbers are 0 .

In this paper the goal is learn of the MLP artificial neural networks to maximize the recognition accuracy of Kurdish letters in training and testing stages of the MLP.

\section{Proposed Model}

In the whole artificial neural networks, the parallel process systems are which are used for recognition of the complex patterns among the data. In fact an artificial neural network is an information processing system which includes some common specifications with the biological neural networks. So, each artificial neural network is formed by a set of the neurons holding a special arrangement. The basic parts of an artificial neural network are the neurons and the communication lines among them. The neurons are

Table 3. Alphabet Matrix creation

Alphabet=[LetterA, LetterB, LetterC, LetterÇ, LetterD, LetterE, LetterÊ, LetterF, LetterG, LetterH, LetterI, LetterÎ, LetterJ, LetterK, LetterL, LetterM, LetterN, LetterO, LetterP, LetterQ,

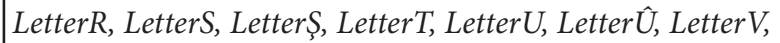
LetterW, LetterX, LetterY, LetterZ]

Table 4. The Target Matrix in $31 * 31$ dimensions

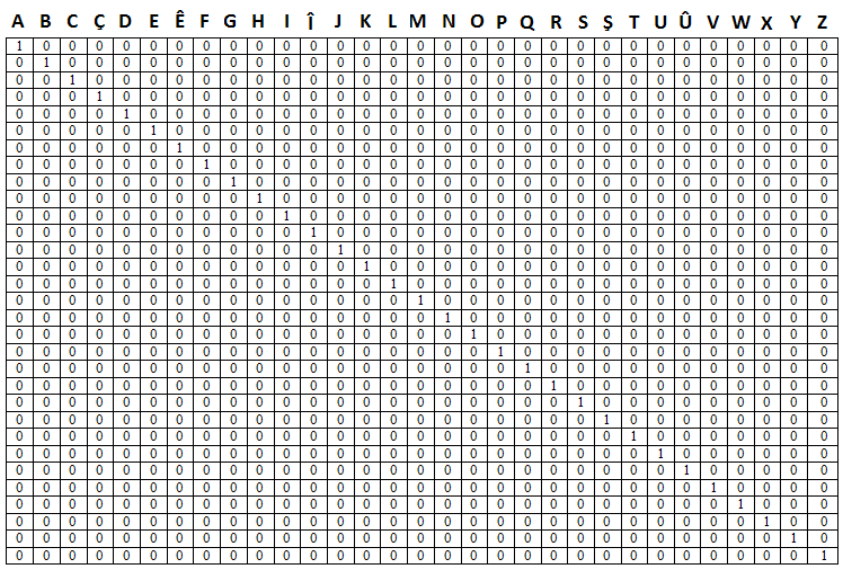

the interlinked processing elements which work together to solve a problem ${ }^{27-29}$. The artificial neural networks are able to learn. This learning takes place via experience and the generalizability for solving the new problems which make this method better than the others ${ }^{30,31}$.

The artificial neural networks made of one neuron are so limited. One of the limitations is that these networks are not able to implement the non-linear functions. The MLP artificial neural networks include the two types of single layer and multi-layer. The single layer perceptron artificial neural networks is only able to learn the problems which are linearly separable, but there are many problems which are not linearly separable ${ }^{32}$ and Kurdish letters recognition is one of them. MLP artificial neural networks are the multi-layer model which can be used for recognition of the Kurdish letters which is a non-linear problem and simply answers. MLP artificial neural networks include one input layer, several hidden layers and one output layer in any of them some neurons could be placed.

In each hidden and output layers the transfer functions are used. The transfer functions are used to determine neuron features to solve different problems ${ }^{33}$. In this paper the logsis transfer function is used both in hidden and output layers. The reason of selecting the logsis transfer function for the both layers (hidden and output layers) is that the output of the both layers fall in the zero and one limits. In other words, as all used Kurdish letters holds matrixes of one and zero values, so it is better to use the Logsis transfer function which is in the range of zero and one. Figure 3 shows the logsis transfer function.

According to the Figure 3 , the logsis function generates the output neuron between one and zero and the formula 1 is used for this function.

$$
a(n)=\frac{1}{1+e^{-n}}
$$

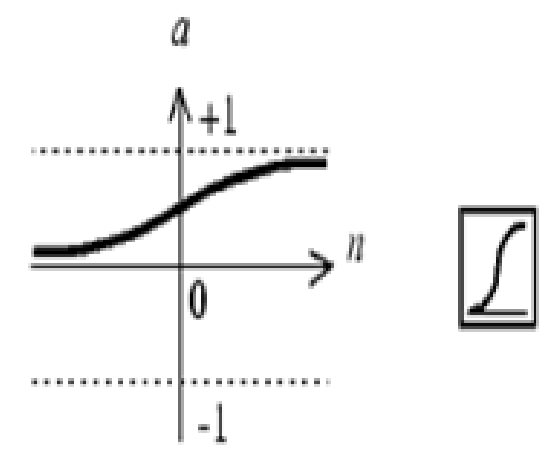

Figure 3. The architecture of the logsis transfer function. 
In this paper, for training of MLP artificial neural networks and correction of the weights for improvement of the recognition accuracy, the back-propagation algorithm is used. In the back-propagation algorithm the updating of any weight takes place via formula 2 .

$$
\Delta w_{j i}=\lambda \delta_{j} o_{i}
$$

According to the formula 2 in which $\lambda$ is the learning rate and if the $\delta 1$ is the output layer, then it is calculated using the formula 3 .

$$
\delta_{j}=o_{j}\left(1-o_{j}\right)\left(t_{j}-o_{j}\right)
$$

Formula 3 calculates the new weights for the output layer and if $\delta 1$ is the hidden layer, then it is calculated using the formula 4 .

$$
\delta_{j}=o_{j}\left(1-o_{j}\right) \sum_{k} \delta_{k} w_{k j}
$$

And the formula 4 calculates the new weights for the hidden layer. The MLP artificial neural networks in this paper have 77 inputs according to the $77^{*} 1$ matrixes, and any of the $77^{*} 1$ matrixes are related to one of the Kurdish letters. As noted before, the target vector includes $31^{*} 31$ matrixes as noted before. In this paper, the goal is to train MLP artificial neural networks to get 77 inputs related to each feature of the letter, proper output from between the ideal output 31 due to $31^{*} 31$ target matrixes determine. The total scheme of the MLP artificial neural networks for recognition of the Kurdish letters is based on Figure 4.

In architecture of Figure 4 it is seen there are 77 inputs related to each letter. The number of the neurons in output layer is 31 and this is because there are 31 Kurdish letters and the number of the hidden layers is considered by experience 5 to get better results and the first hidden layer holds 6 neurons, the second hidden layer holds 7 neurons, the third hidden layer holds 8 neurons, the fourth hidden layer holds 9 neurons and the fifth hidden layer holds 10 neurons. Also according to the Figure 4, the transfer function for neurons of the hidden and output layers is logsig. The structure of the implementation in Matlab environment is based on newpr function ${ }^{4}$ and newpr function is used for defining the MLP artificial neural networks.

According to the Alphabet matrix which have $77^{*} 31$ dimensions and all the letters are placed in this matrix, $85 \%$ is selected for training and the $15 \%$ for testing and the Table 5 shows this Division.

In this paper, according to the Table 5 the accuracy of the recognition of the Kurdish letters is showed in training and testing stages. To get the recognition accuracy at training and testing stages, the output of the MLP of training and testing of the data and the target matrix are joined to each other column by column. Then the values of the rows and columns are divided into three groups and if the value of any row and column was zero, the value would be

Table 5. The division of the data for training and testing

\begin{tabular}{lcl}
\hline Sample numbers & Percent & Type \\
\hline 65 & $85 \%$ & Train \\
12 & $15 \%$ & Test \\
77 & $100 \%$ & Total \\
\hline
\end{tabular}

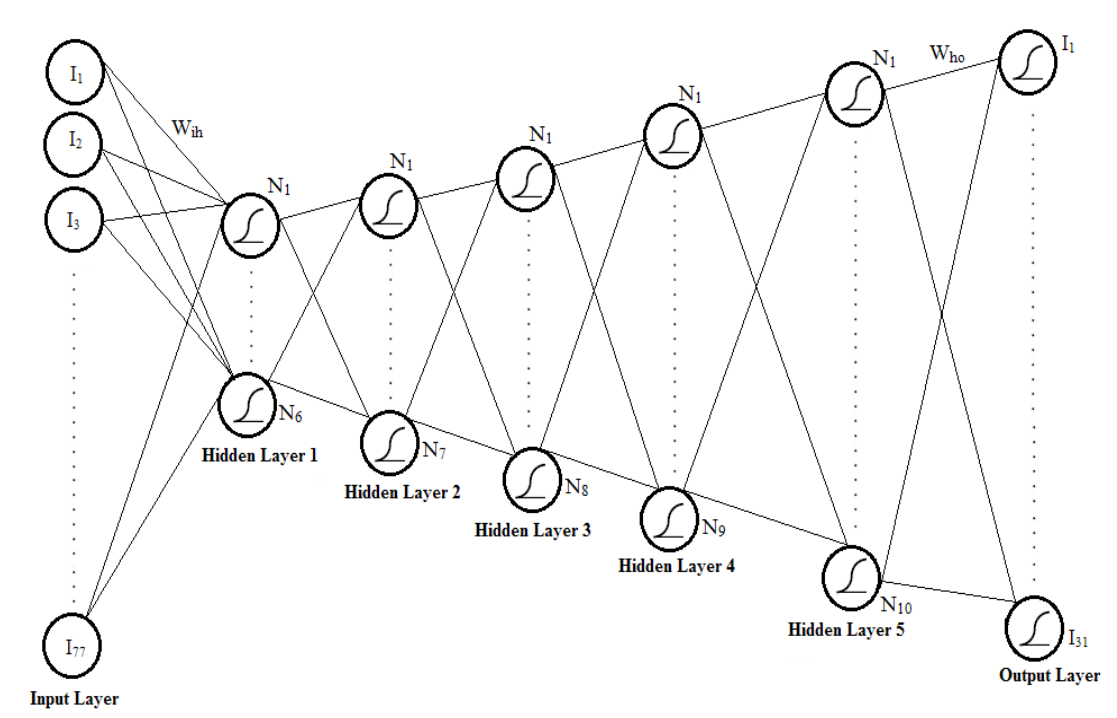

Figure 4. The proposed scheme for MLP artificial neural networks in recognition of the Kurdish letters. 
zero and if the value was more than zero it would be 1 and at last if the value was less than zero it would be -1 . Then all values of each column is summed up to reach a definite value and then the numbers of the values of summing up the each column which were zero are obtained and then the formula 5 will be used for reaching this recognition accuracy.

$$
\begin{aligned}
\text { Accuracy }= & (\text { length }(\text { target })-\text { length }(\text { find }(S==0))) / \\
& \text { length }(\text { target }) * 100
\end{aligned}
$$

According to the formula $5, \mathrm{~S}$ is a matrix in which the resulted values of adding each column equal to 0 placed in it. In this formula we have subtracted the length of the target matrix from the numbers which are equal to 0 in $S$ matrix and we do this because the number 1 in any letter matrix show the letter and there is no need for numbers 0 and they are just extra load in the memory. The reason of selecting number 100 is that the accuracy of the recognition in training and testing stages is calculated $100 \%$.

According to the explanation about designing the MLP artificial neural networks, Figures 5 and 6 show the Kurdish letters recognition accuracy in training and testing stages after execution of MLP artificial neural networks, respectively.

According to the Figures 5 and 6 as it can be seen, the accuracy of Kurdish letters recognition is $85.1535 \%$ in training stage and $81.2677 \%$ in testing stage. The points which are colored black in Figures 5 and 6 are the matrix Target. According to Figures 5 and 6, if these black points are covered by the blue points which are the output of the

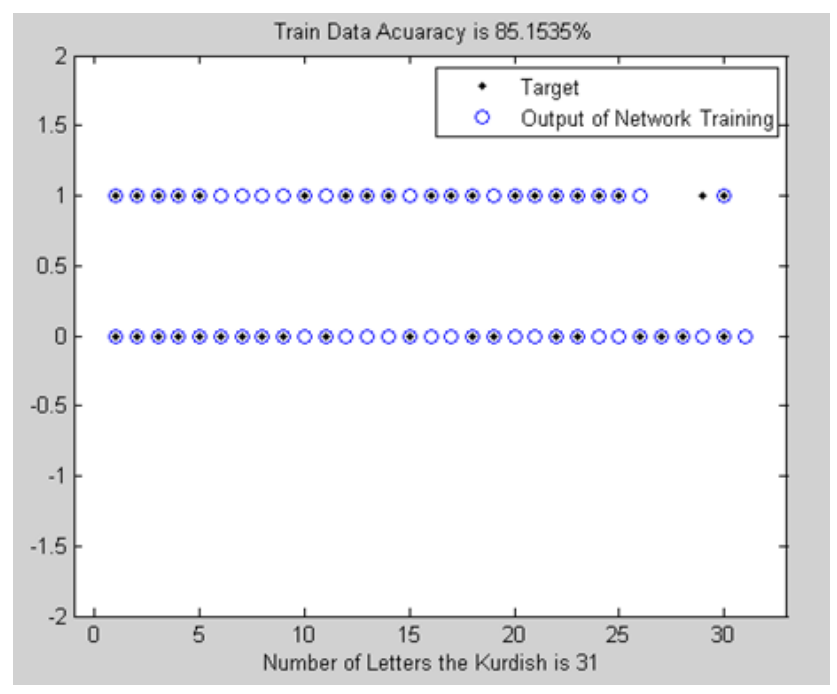

Figure 5. Kurdish letters recognition accuracy in training stage of MLP artificial neural networks. artificial neural networks MLP in training and testing stages, it will mean that this coverage is recognition of matrix Target using output of artificial neural networks MLP in training and testing stages, and this recognition is right. If some of the black points are not covered by the blue points, it will mean that the blue points (the output of the MLP artificial neural networks) could have not recognize these black points (matrix Target) right. The points zero and one in a vertical axis are the values of the Kurdish letters in matrix Target and as said before each letter is formed by zero and one values. The horizontal axis includes 31 Kurdish letters and these letters are shown in Figures 5 and 6 in form of the black colored points of matrix Target.

So, as it is clear from recognition accuracy in training and testing stages, MLP artificial neural networks hold high accuracy in recognition of Kurdish letters.

\section{Conclusions and the Future Works}

Automation of the reading texts has been very important in different languages and presenting a method for this field with high accuracy has been the subject of the researches. Automation of reading the texts in different languages leads to save time and costs. The letters recognition is one of the subjects which have been very important for automation of text reading point of view and many methods are used to implement the letters recognition systems.

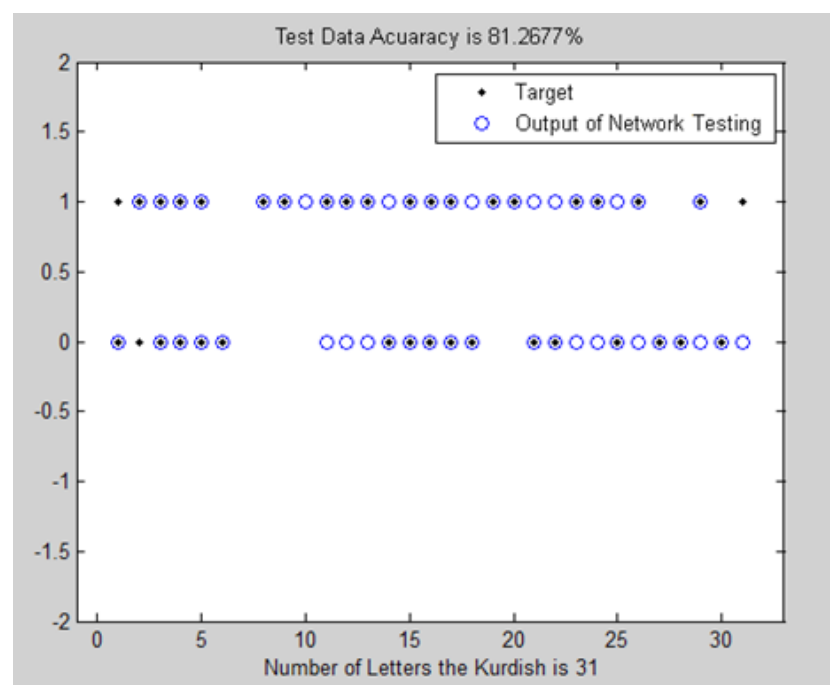

Figure 6. Kurdish letters recognition accuracy in testing stage of MLP artificial neural networks. 
As the presented methods have shortages in many fields, it is important to use a new and general method for recognition of the letters which is not specific to a special language and is generalizable to all languages. So the artificial neural networks are very strong in patterns recognition in comparison to other methods. In this paper it is tried to use the MLP artificial neural networks to present a method for recognition of the Latin-Kurdish letters which contain 31 letters. Although, seriously has not been provided the possibility of recognizing this letters by using the different models of artificial neural networks. In this paper, efficiency factor for recognition of the Kurdish letters is to maximize the recognition accuracy of the Kurdish letters in training and testing stages. This recognition accuracy rate is $85.1535 \%$ in training stage and $81.2677 \%$ in testing stage. The proposed model in this paper for all languages with Latin-letters is usable. The works which could be done in future are to use the other models of artificial neural networks like PNN and Generalized regression neural networks to recognize the Kurdish letters and it is also possible to compare any of these artificial neural networks to the MLP artificial neural network or any other artificial neural network to understand which one has the better accuracy of the Kurdish letters recognition.

\section{References}

1. Kang M, Palmer-Brown D. A modal learning adaptive function neural network applied to handwritten digit recognition. Inform Sci. 2008; 178(20):3802-12.

2. Lee S-W. Multilayer cluster neural network for totally unconstrained handwritten numeral recognition. Neural Network. 1995; 8(5):783-92.

3. Palmer-Brown D, Kang MD: An adaptive function neural network. The 7th International Conference on Adaptive and Natural Computing Algorithms (ICANNGA05); 2005; Coimbra, Portugal; Springer Vienna; p. 1-4.

4. Demuth H, Beale M. Neural network toolbox for use with MATLAB: User's Guide. Version 4. The MathWorks, Inc. 3 Apple Hill Drive Natick, MA; 2002.

5. Drucker H, Schapire R, Simard P. Boosting performance in neural networks. Int J Pattern Recogn Artif Intell. 1993; 7(4):61-75.

6. Al-Omaria FA, Al-Jarrah O. Handwritten Indian numerals recognition system using probabilistic neural networks, Adv Eng Informat. 2004; 18(1):9-16.

7. Gharehchopogh FS, Ahmadzadeh E. Artificial neural network application in letters recognition for Farsi/ Arabic manuscripts. International Journal of Scientific \& Technology Research. 2012; 1(8):49-54.
8. Gharehchopogh FS. Neural network application in software cost estimation: a case study. 2011 International Symposium on Innovations in Intelligent Systems and Applications (INISTA 2011); 2011 Jun 15-18. Istanbul, Turkey: IEEE. p. 69-73.

9. Sibanda W, Pretorius P. Novel application of Multi-Layer Perceptrons (MLP) neural networks to model HIV in South Africa using Seroprevalence data from antenatal clinics. Int J Comput Appl Tech. 2011; 35(5):26-31.

10. Lyon R, Yaeger L. On-line hand-printing recognition with neural networks. Proceedings of Fifth International Conference on Microelectronics for Neural Networks; 1996 Feb 12-14; Lausanne. p. 201-12.

11. Gao W. New evolutionary neural networks. International Conference on Neural Interface and Control. 2005 May 26-28. p. 167-71.

12. Ahangar RG, Ahangar MF. Handwritten Farsi character recognition using Artificial Neural Network. Int J Comput Sci Inform Secur. 2009; 4(1-2).

13. Almualim H, Yamaguchi S. A method for recognition of Arabic cursive handwriting. IEEE T PATTERN ANAL. 1987; 9(5):715-22.

14. Abuhaiba ISI, Mahmoud SA, Green RJ. Recognition of handwritten cursive Arabic characters. IEEE T Pattern Anal. 1994 Jun; 16(6):664-72.

15. Morasso P. Neural models of cursive script handwriting. International Joint Conference on Neural Network (IJCNN); 1989; Washington, DC, USA. p. 539-42.

16. Smith SJ, Baurgoin MO, Sims K, Voorhees HL. Handwritten character classification using nearest neighbor in large database. IEEE T Pattern Anal. 1994; 16(10):915-19.

17. Sitamahalakshmi T, Babu AV, Jagadeesh M, Mouli KVVC. Performance comparison of radial basis function networks and probabilistic neural networks for Telugu character recognition. Global J Comput Sci Tech. 2011; 11(4):8-16.

18. Verma BK. Handwritten Hindi character recognition using multilayer perceptron and radial basis function neural networks. IEEE, International Conference on neural network; 1995 Nov/Dec; Perth, WA. p. 2111-15.

19. Sureshkumar C, Ravichandran T. Handwritten Tamil character recognition and conversion using neural network. Int J Comput Sci Eng. 2010; 2(7):2261-67.

20. Yeung DS. A neural network recognition system for handwritten Chinese character using structure approach. 1994 IEEE International Conference on Neural Networks, 1994. IEEE World Congress on Computational Intelligence; 1994 Jun 27-Jul 2; Orlando, FL. p. 4353-58.

21. Prochazka, AP. Feed-forward and recurrent neural networks in signal prediction. IEEE, In 4th International Corference on Computational Cybernetics; 2007. p. 93-6.

22. Bahmani Z, Alamdar F, Azmi R, Haratizadeh S. Off-line Arabic/Farsi handwritten word recognition using RBF 
neural network and genetic algorithm. IEEE International Conference on Intelligent Computing and Intelligent Systems (ICIS), Xiamen; 2010. p. 352-57.

23. Ashok J, Rajan EG. Writer identification and recognition using radial basis function. Int J Comput Sci Inform Tech. 2010; 1(2):51-7.

24. Kochari JA, Mohabadi A. Letter recognition using fuzzy logic, Second International Conference of Information Technology. (Language: Farsi).

25. Chiang JH. A hybrid neural network model in handwritten word recognition. Neural Network. 1998; 11(2):337-46.

26. Jaberian S. Letter recognition using pen movement for Farsi manuscript; 1997. (Language: Farsi).

27. Mcinerney M, Dhawan A P. Use of genetic algorithms with back propagation in training of feed-forward neural networks. IEEE International Conference on Neural Neworks; 1993; San Francisco, USA. p. 203-08.

28. Le Cun Y, Boser B, Denker JS, Henderson D, Howard RE, Hubbard W, Jackel LD, Baird HS. Constrained neural network for unconstrained handwritten digit recognition. In Proceedings of lst Internatinonal Workshop on Frontiers in Handwriting Recognition. 1990; Montreal, Canada. p. 145-54.

29. Zebardast B, Ghaffari A, Masdari M. A new generalized regression artificial neural networks approach for diagnosing heart disease. Int J Innovat Appl Stud. 2013; 4(4):679-89.

30. Yamada K, Kami H, Temma T, Tsukumo J. Handwritten numeral recognition by multilayered neural network with improvedlearning algorithm. International JointConference on Neural Networks (IJCNN). 1989; Washington, DC. p. 259-66.

31. Liu J, Gader P. Neural networks with enhanced outlier rejection ability for off-line handwritten word recognition. Pattern Recogn. 2002; 35(10):2061-71.

32. Das N, Mollah AF, Saha S, Haque SS. Handwritten Arabic numeral recognition using a multi-layer perceptron. Proceedings National Conference on Recent Trends in Information Systems (ReTIS-06); 2006; Kolkata, India. p. 200-03.

33. Er O, Yumusak N, Temurtas F. Chest diseases diagnosis using artifical neural networks. Expert Syst Appl. 2010; 37(12):7648-55. 